\title{
Experimental Investigation of Surface Plasmon Resonance Using Tapered Cylindrical Light Guides with Metal-Dielectric Interface
}

\author{
Sharmila Ghosh, Kaushik Brahmachari, Mina Ray \\ Department of Applied Optics \& Photonics, College of Science \& Technology, \\ University of Calcutta, Kolkata, India \\ Email: mraphy@caluniv.ac.in
}

Received November 11, 2011; revised December 6, 2011; accepted January 5, 2012

\begin{abstract}
In this paper we report an experimental investigation on the observation of surface plasmon resonance at the metaldielectric interface of silver coated tapered light guiding glass rods of different dimensions. Chemical deposition technique has been used for metallic deposition of silver on the Tapered glass rod. Angular interrogation in Kretschmannlike configuration of this non-planar (cylindrical) structure is used to locate the resonance dip in the reflectance measurement. Fabrication simplicity, in addition to the inherent advantage of using different types of tapered structures, makes this study important in the development of nanoplasmonics. The experimental study is accompanied by some interesting results.
\end{abstract}

Keywords: Glass Waveguides; Thin Films; Plasmonics; Surface Plasmons

\section{Introduction}

The Surface plasmon resonance (SPR) phenomenon is one of the most useful areas of research in the field of Nanotechnology/Nanophotonics. Surface plasmons are the collective charge oscillations at metal-dielectric interface [1]. Surface plasmon resonance phenomena has lots of applications in medicine, Nanobiotechnology, environmental monitoring etc [2]. The earlier studies relating to SPR phenomenon employed either Kretschmann [3] or Otto configuration [4]. Surface plasmon resonance phenomenon is an important tool used for developing sensors. The first SPR sensor was developed for gas detection and biosensing [5,6]. Many researchers have reported their work on SPR-based fiber optic sensor [7-13].

Almost all the methodology used in above literature involve sophisticated instrumentation as far as fabrication of the actual nano-thickness multilayered structured is concerned. In most of the methods complex planar structures are used, which are though simpler to analyze mathematically but need a high level of accuracy in fabrication and measurements. Earlier, Ray et al. have published the SPR based theoretical [14-16] as well as experimental work $[17,18]$ where main focus was on the development of novel methodology as well as reduction of complexity in the structure.

In the present work we have investigated surface plasmon resonance phenomenon experimentally using two different kind of tapered glass rods made of fused silica material. A thin layer of silver having thickness of the order of $50 \mathrm{~nm}$ has been chemically deposited over a specified length of the external surface of these rods. Effect of varying the sensing length has also been investigated.

\section{Theory}

\subsection{Basic Theory of Surface Plasmon Resonance}

The excitation of surface plasmons at metal/dielectric interface results in the transfer of energy from incident light to surface plasmons, which reduces the intensity of the reflected light. Surface plasmons are coherent electron oscillations at the interface between any two materials (like metal/dielectric) where real part of dielectric function changes sign across the interface. A medium of large negative dielectric constant is a good selection to observe surface plasmon resonance phenomenon. As a result, most noble metals such as aluminium, gold and silver can exhibit resonant absorption by surface plasmon excitation. When a p-polarized light beam (having direction of vibration parallel to the plane of incidence) is incident from a light coupling device like glass/epoxy to a noble metal having large negative dielectric constant and as light is going from a higher refractive index medium (glass/epoxy) to a lower refractive index medium 
(metal), an evanescent wave is created at the glass-metal interface due to the slight penetration of the incoming light wave into the metal layer having thickness of the order of nanometer. As a result, there will be energy coupling between evanescent wave and surface plasmon wave at the glass-metal interface. These surface plasmon waves are created due to surface electrons on the glass metal interface. When the wave vector of the evanescent wave $\left(k_{e v}\right)$ and surface plasmon wave $\left(k_{S P}\right)$ matches then entire amount of evanescent wave energy is transferred to the surface plasmon wave and due to resonance the reflected light intensity from the output beam will be minimized. Now the phase matching condition needed for surface plasmon resonance is given by [3]

$$
\left(k_{e v}\right)=\left(k_{S P}\right)
$$

where

$$
\begin{aligned}
& k_{e v}=\frac{\omega}{c} \sqrt{\varepsilon_{p}} \sin \theta \\
& k_{S P}=\frac{\omega}{c}\left(\frac{\varepsilon_{m} \varepsilon_{s}}{\varepsilon_{m}+\varepsilon_{s}}\right)^{1 / 2}
\end{aligned}
$$

where $\varepsilon_{p}$ represents the dielectric constant of the material of the coupling device like (glass/epoxy), $\varepsilon_{m}$ represents the dielectric constant of the metal layer, $\varepsilon_{s}$ represents dielectric constant of the sensing layer, $\theta$ is the angle of incidence of the beam, $\omega$ is the frequency of the incident light beam and $\mathrm{c}$ is the velocity of light.

The excitation of surface plasmons occurs when the wave vector of evanescent wave exactly matches with that of surface plasmons of similar frequency. This occurs at a particular angle of incidence $\theta_{\text {res }}$, which is known as SPR angle. Thus the resonance condition for surface plasmon resonance is given by

$$
\frac{\omega}{c} \sqrt{\varepsilon_{p}} \sin \theta_{r e s}=\frac{\omega}{c}\left(\frac{\varepsilon_{m} \varepsilon_{s}}{\varepsilon_{m}+\varepsilon_{s}}\right)^{1 / 2}
$$

\subsection{Reflectance for a 3-Layer Kretschmann Configuration}

Fresnel reflection formulae for 3-layer model of conventional Kretschmann configuration of the prism (subscript-pr)/metal (subscript-1)/air (subscript-2) system can be summarized as follows. These formulae have been generally used for theoretical simulation of planar geometry and we have made necessary modifications for the non-planar structures under consideration.

The reflectance $R$, for $\mathrm{p}$-polarization is given by

$$
R=\left|r_{p r 12}^{p}\right|^{2}=\left|\frac{\left(r_{p r 1}^{p}+r_{12}^{p} \exp \left(2 i k z_{1} d_{1}\right)\right)}{\left(1+r_{p r 1}^{p} r_{12}^{p} \exp \left(2 i k z_{1} d_{1}\right)\right)}\right|^{2}
$$

where

$$
\begin{gathered}
r_{i k}^{p}=\frac{\left(n_{k} \cos \theta_{i}-n_{i} \cos \theta_{k}\right)}{\left(n_{k} \cos \theta_{i}+n_{i} \cos \theta_{k}\right)} \\
r_{12}^{p}=\frac{\left[\left(\sqrt{\varepsilon_{1}-n_{p r}^{2} \sin ^{2} \theta_{p r} / \varepsilon_{1}}\right)-\left(\sqrt{n_{2}^{2}-n_{p r}^{2} \sin ^{2} \theta_{p r} / n_{2}^{2}}\right)\right]}{\left[\left(\sqrt{\varepsilon_{1}-n_{p r}^{2} \sin ^{2} \theta_{p r} / \varepsilon_{1}}\right)+\left(\sqrt{n_{2}^{2}-n_{p r}^{2} \sin ^{2} \theta_{p r} / n_{2}^{2}}\right)\right]}
\end{gathered}
$$

phase factor $=k z_{1} d_{1}=k_{1} d_{1} \cos \theta_{1}$

$$
=\left(\frac{2 \pi}{\lambda}\right) d_{1}\left(\sqrt{\left(\varepsilon_{1}-n_{p r}^{2} \sin ^{2} \theta_{p r}\right)}\right)
$$

\subsection{Mathematical Formulation for Taper Light Guide}

Figure 1 depicts the typical ray path within a taper light guide under attenuated total internal reflection (ATIR) mode. For linear tapering, $\rho(z)$ is the taper radius at a distance " $z$ " from input end of the taper. Angle of incidence $\theta_{i}$ of the ray at input end of the taper will be transformed into incident angle $\varphi(z)$ at the taper sensing region located at a distance $\mathrm{z}$ from the input end.

If $n_{\text {air }}$ is the refractive index of air and $n_{g}$ is the refractive index of the taper light guide material (i.e. glass), then using Snell's law,

$$
n_{\text {air }} \sin \theta_{i}=n_{g} \sin \theta_{r}
$$

$\theta_{i}$ is the angle of incidence controlled by the rotating table. By knowing $\theta_{i}$ and using Equation (9), we can determine the value of $\theta_{r}$. Assuming $\theta=\left(90-\theta_{r}\right)$, the angle $\varphi(z)$ can be calculated as

$$
\varphi(z)=\cos ^{-1}\left[\frac{\rho_{i} \cos \theta}{\rho(z)}\right]-\tan ^{-1}\left(\frac{\rho_{i}-\rho_{o}}{L}\right)
$$

where

$$
\rho(z)=\rho_{i}-\frac{z}{L}\left(\rho_{i}-\rho_{o}\right) .
$$

And $\tan ^{-1}\left(\frac{\rho_{i}-\rho_{o}}{L}\right)=\Omega$ represents the taper angle. $\theta_{r}$ is the angle of refraction at the input end, $\rho_{i}$ and $\rho_{o}$ denote the radii at input and output end of the taper respectively and $L$ is the length of the taper [10]. As depicted schematically in Figure 1, we can easily visualize that as the light propagates through the tapered glass rod, the angle of incidence decreases along the glass-air interface of taper outer surface where there is no coating. It is to be noted that when the light beam reaches the sensing area, SPR occurs only at the first reflection on the glass-metal interface of the tapered glass rod provided the phase matching condition of Equation (1) is satisfied. 


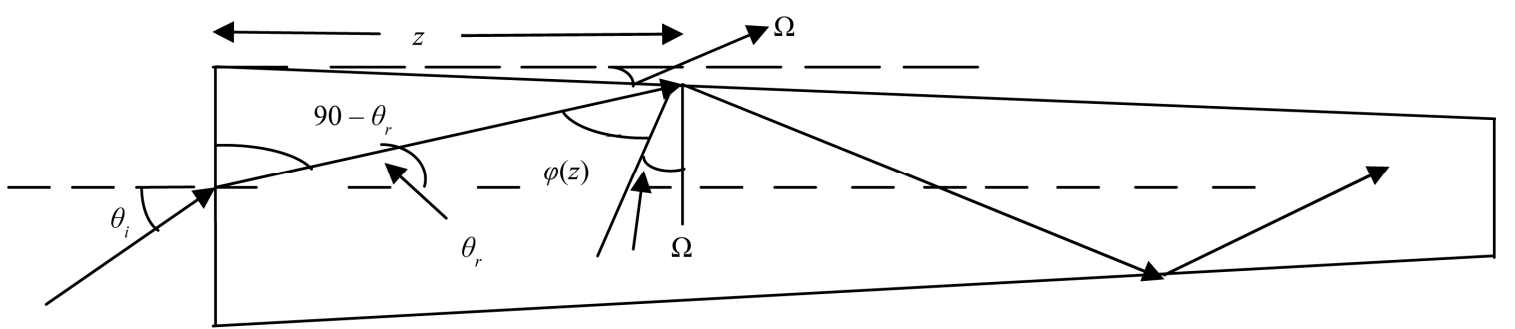

Figure 1. Taper structure used as the light coupling device for SPR sensing.

Consequently, for other successive reflection of the glass-metal interface finally result in the decrease of light intensity measured at the exit end face of the tapered glass rod and one will get the minimum intensity in the output beam. Here the output beam power is measured at the exit end of the taper, which is further normalized by the highest value of the power measured at the output end. As normalized reflectance value at the glass-metal interface is directly proportional to this, we have plotted our results in terms of reflectance vs. angle of incidence.

\section{Sample Preparation}

As shown in Figure 2 and Figure 3, two types of tapers are used for our experimental purpose and corresponding specifications are as shown in Table 1. Main purpose of using different sensing layer length and location is to investigate their effect on the observed SPR. Sensing region is located at $3 \mathrm{~cm}$ from larger diameter and $1.3 \mathrm{~cm}$ from the smaller diameter for Taper- 1 whereas it is located at $3.9 \mathrm{~cm}$ from larger diameter and $2.4 \mathrm{~cm}$ from the smaller diameter for Taper-2.

\subsection{Deposition of Silver Film Using Chemical Deposition Technique}

Chemical deposition technique that we have used for the purpose of coating is described in the self-explanatory Figure 4. It is to be noted that before all the samples are dipped in this mixture of stock solution $\mathrm{A}$ and $\mathrm{B}$, the samples are dipped in $5 \% \mathrm{SnCl}_{2}$ solution prepared with $2.5 \mathrm{gm} \mathrm{SnCl}_{2}$ and $50 \mathrm{cc}$ distilled water. Here $\mathrm{Sn}^{2+}$ ion act as binding agent for deposited $\mathrm{Ag}^{2+}$ ion on the samples. Moreover, by controlling the time of deposition we can control the thickness of the deposited silver film over the samples. Here the time of deposition within the solution is of the order of 120 seconds for both Taper-1 and Taper-2. For this time of deposition the thickness of coated silver film was found to vary within the range of $35 \mathrm{~nm}$ to $38.3 \mathrm{~nm}$ [19]. For simulation of Fresnel's reflection equation with non-planar structures using Equation (10) and Equation (11) it was found that thickness of the silver film of the order of $35 \mathrm{~nm}$ is necessary to obtain surface plasmon resonance with non-planar structures for the Attenuated Total Internal reflection (ATIR) at a particular

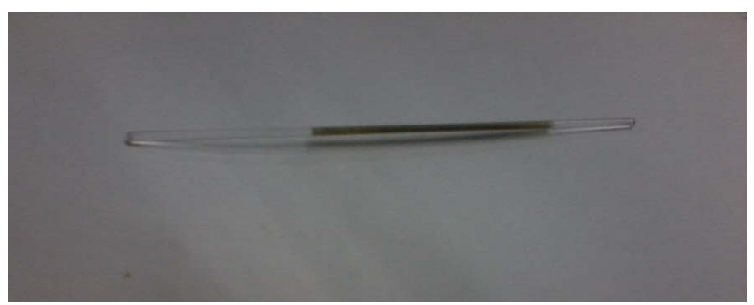

Figure 2. Taper-1.

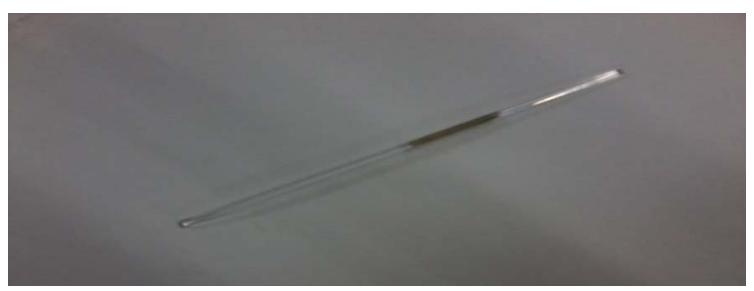

Figure 3. Taper-2.

Table 1. Specifications of two tapers.

\begin{tabular}{ccccc}
\hline Sample & Length & $\begin{array}{c}\text { Sensing region } \\
\text { length }\end{array}$ & $\begin{array}{c}\text { Entrance } \\
\text { diameter }\end{array}$ & $\begin{array}{c}\text { Exit } \\
\text { diameter }\end{array}$ \\
\hline Taper-1 & $8.2 \mathrm{~cm}$ & $3.9 \mathrm{~cm}$ & $3.0 \mathrm{~mm}$ & $2.0 \mathrm{~mm}$ \\
Taper-2 & $7.9 \mathrm{~cm}$ & $1.6 \mathrm{~cm}$ & $3.0 \mathrm{~mm}$ & $2.0 \mathrm{~mm}$ \\
\hline
\end{tabular}

location within the taper. Finally all the samples are dipped in distilled water for 30 minutes and then dried to ensure that the surface is free from any water mark.

\subsection{Protection of the Silver Coating from Oxidization}

Though silver is a good candidate for observation of SPR, it is prone to oxidization. In order to protect the deposited silver film from getting oxidized, a mixture of $2 / 3$ part $99 \%$ pure tetra ethyl orthosilicate $\left[\mathrm{Si}\left(\mathrm{OC}_{2} \mathrm{H}_{5}\right)_{4}\right]$ and $1 / 3$ part ethyl alcohol $\left[\mathrm{C}_{2} \mathrm{H}_{5} \mathrm{OH}\right]$ can be used. When the samples are dipped in this mixture for a short duration and heated to $250^{\circ} \mathrm{C}$ in vacuum oven, a thin layer of $\mathrm{SiO}_{2}$ so created will protect the silver film from oxidization. It is to be noted that samples should be dipped within the solution for $1 \mathrm{sec}$ only in order to ensure the thickness of the $\mathrm{SiO}_{2}$ protective layer of the order of $1 \mathrm{~nm}$ to $5 \mathrm{~nm}$.

$$
\mathrm{Si}\left(\mathrm{OC}_{2} \mathrm{H}_{5}\right)_{4} \rightarrow \mathrm{SiO}_{2}+2 \mathrm{O}\left(\mathrm{C}_{2} \mathrm{H}_{5}\right)_{2}
$$




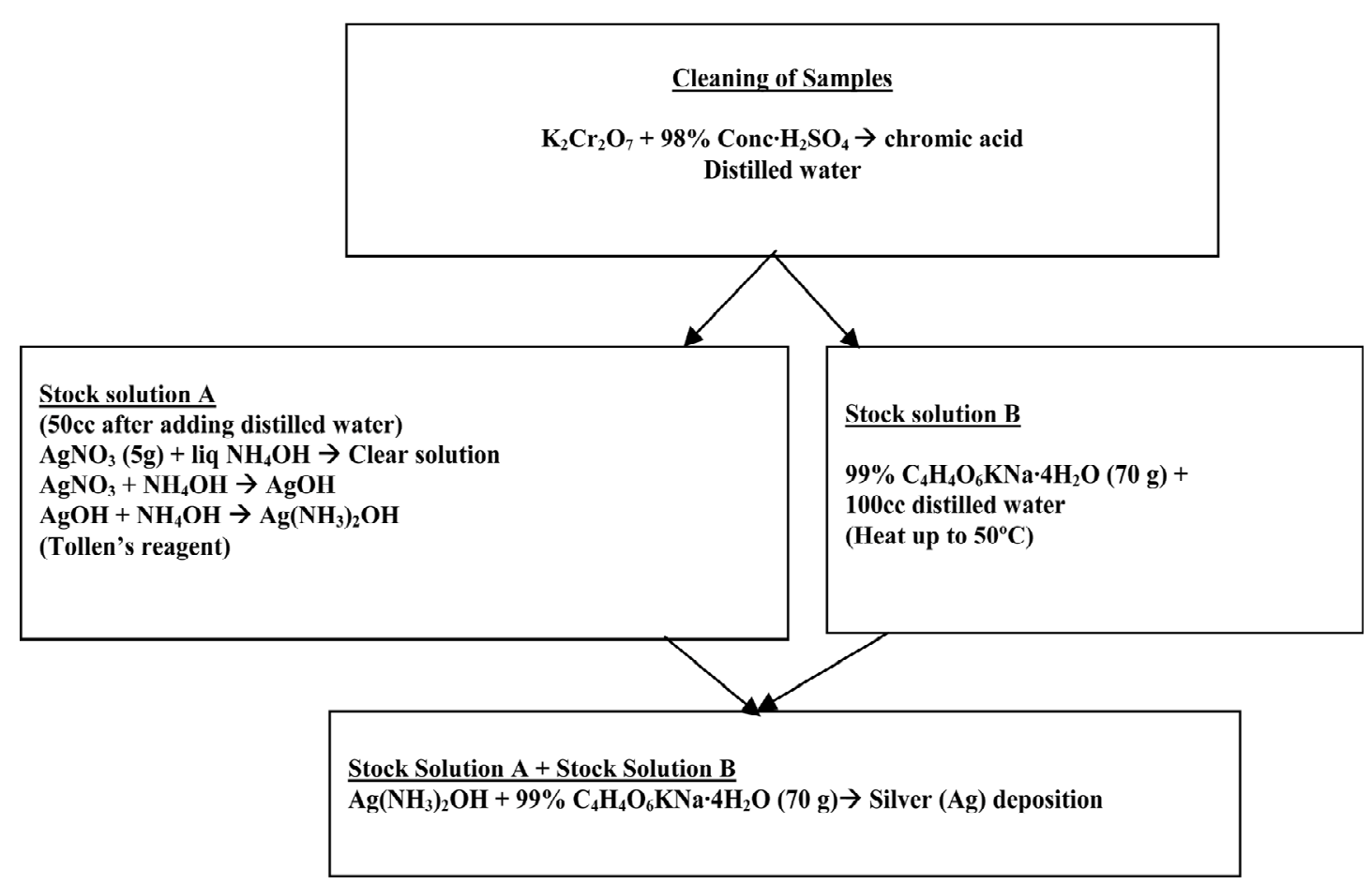

Figure 4. Chemical silver deposition process.

\section{Experimental Arrangement}

Our basic experimental set-up for the angular interrogation reflectance measurement is shown in Figures 5(a) and (b). The He-Ne laser is used as the source of electromagnetic radiation to excite the plasmon. A polarizer is used to make the input beam p-polarized so that its direction of vibration becomes parallel to the plane of incidence to excite the surface electrons of the metal. Angle of interrogation mode is realized by placing the experimental samples on the rotating table in such a manner so as to coincide the input end of the tapered rod with the rotating axis of this table. The output beam from the tapered rod is detected by a photo detector and measured by a power meter.

\section{Detail Specification of Experimental Components Used}

Laser source: Melles Griot, $5 \mathrm{mw}$ at $632.8 \mathrm{~nm}$. Class 3R laser product IEC.

Polarizer: Yolux, India make, sheet polarizer.

Rotating table: Newport rotating table.

Photo detector: PIN photo detector, Sensor oriel Detection system Model 7072, SER NO 412, ORIEL CORPORATION, STANFORD, CONN-06902. I/P, 110 volts, frequency $=50 / 60 \mathrm{~Hz}$, Current $=0.5 \mathrm{amp}$.

\section{Results and Discussions}

For Taper-1, from the Figure $\mathbf{1}$ it can be shown that as

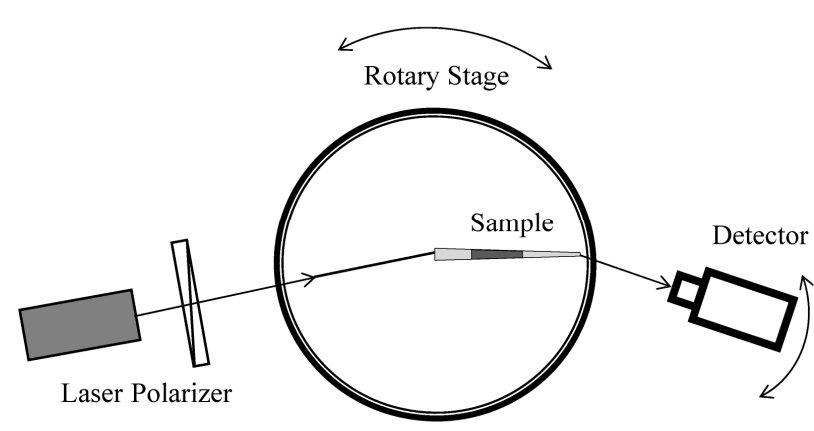

(a)

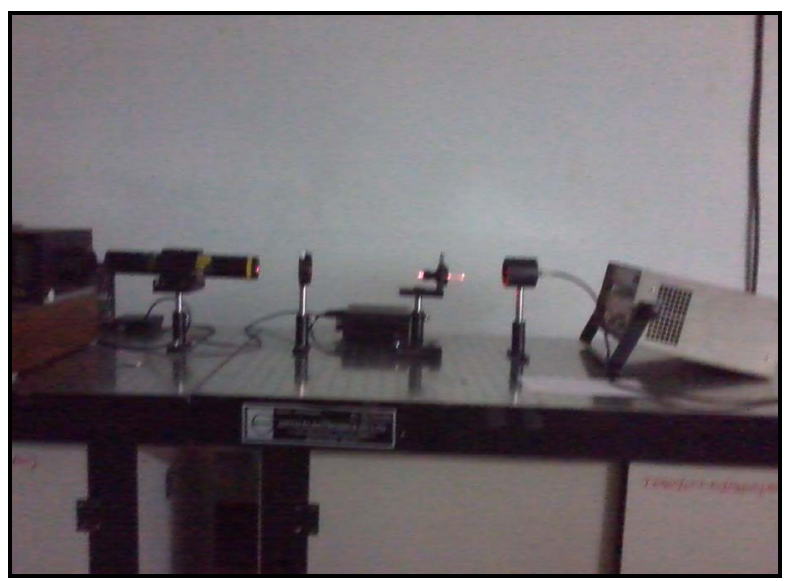

(b)

Figure 5. (a) Experimental set-up (schematic); (b) Experimental set-up (laboratory). 
the angle of incidence $\theta_{i}$ varies at the input end of the taper, angle of incidence $\varphi(z)$ at the sensing region varies according to the formulae given by Equation (9), Equation (10). Thus, when $\theta_{i}=56^{\circ}, \theta_{r}=35^{\circ}$,

$\theta=\left(90-\theta_{r}\right)=55^{\circ}$. Which gives $\varphi(3.0)=45.71^{\circ}$ which is the SPR angle of fused silica-silver interface having air as the sensing region. Here taper angle for Taper-1 is $\Omega=3.489^{\circ}$ as found from sec 2.3 and at the point of first reflection at the sensing region, taper radius is $\rho(z)=1.317 \mathrm{~cm}$ as found from Equation (11). Knowing taper angle $\Omega$, taper radius $\rho(z)$ and angle of incidence $\varphi(z)$ at the sensing region and using Fresnel's reflection equation we can simulate the angle of incidence vs. reflectance graph considering the first point of reflection of the sensing region of non-planar tapered geometrical structures. Temperature during the experiment was fixed to $22^{\circ}$ centrigrade.

Geometry of Figure 1 suggests that after few reflections, the light might fall on the reflective surface almost perpendicularly due to the fact that as light propagates along the length of taper, the angle of incidence decreases for each successive reflection on the surface of the glass rod. But we have restricted the length of our sample as shown in Table 1, so as to avoid the occurrence of such a situation. Moreover, as $35 \mathrm{~nm} \mathrm{Ag}$ layer is semi-transparent and act as gray-filter, light may escape the rod sideways. But as the angle of incidence at the taper glass rod is kept greater than the critical angle, total internal reflection (TIR) is ensured, although there is some loss due to refraction at the uncoated glass when wave propagates further.

Similarly for Taper-2, taper angle is $\Omega=3.621^{\circ}$ and at the point of first reflection at the sensing region taper radius is $\rho(z)=1.253 \mathrm{~cm}$. When $\theta_{i}=52^{\circ}, \theta_{r}=33^{\circ}$, $\theta=\left(90-\theta_{r}\right)=57^{\circ}$ which gives $\varphi(3.9)=45.69^{\circ}$, which is the SPR angle of fused silica-silver interface having air as the sensing medium. Here taper angle for Taper-2 is $\Omega=3.621^{\circ}$ as found from sec 2.3 and at the point of first reflection at the sensing region, taper radius is $\rho(z)=1.253 \mathrm{~cm}$ as found from Equation (11). Knowing taper angle $\Omega$, taper radius $\rho(z)$ and angle of incidence $\varphi(z)$ at the sensing region and using Fresnel's reflection equation we can simulate the angle of incidence vs. reflectance graph considering the first point of reflection of the sensing region of non-planar tapered geometrical structures. Temperature during the experiment was fixed to $22^{\circ}$ centrigrade.

From Figure 6 and Figure 7, it is clear that simulation results have good agreements with that of experimental results. Though Taper-1 and Taper-2 have different sensing region lengths, there is no significant effect on the experimental results for SPR curve, because for these two tapers, SPR is happening only for the first reflection of the glass-metal interface of the tapered glass rod

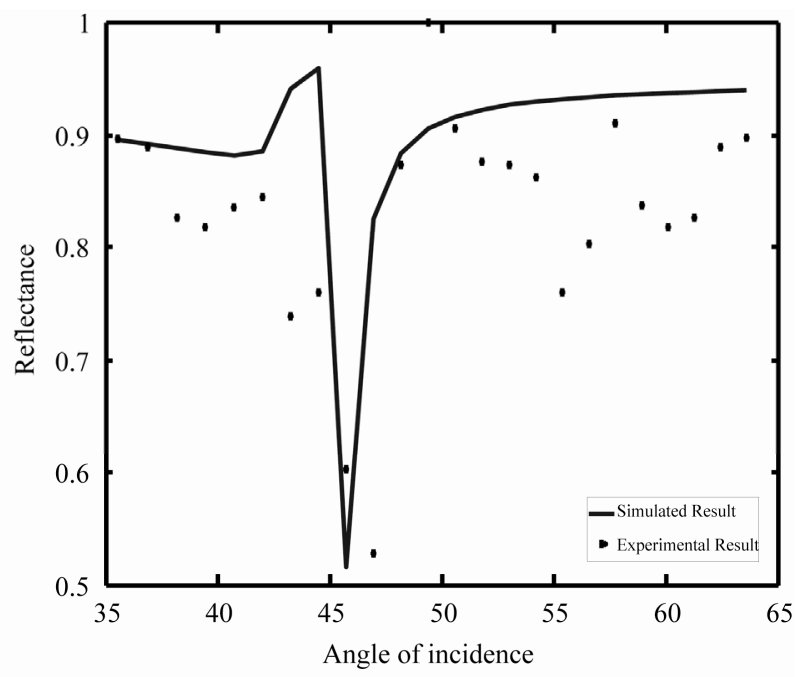

Figure 6. Surface Plasmon Resonance curve showing the variation of reflectance with angle of incidence at the fused silica-silver interface of Taper-1 having air as sensing medium.

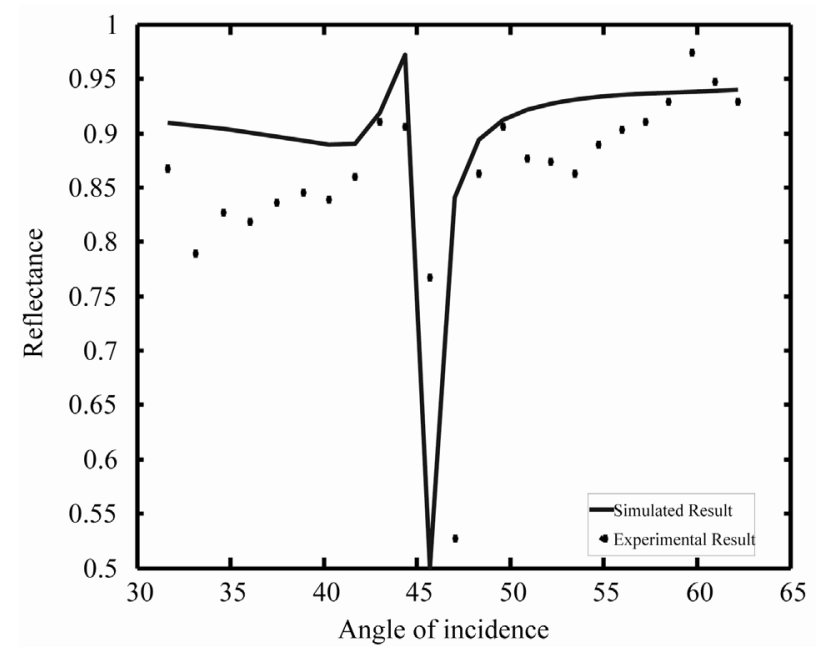

Figure 7. Surface Plasmon Resonance curve showing the variation of reflectance with angle of incidence at the fused silica-silver interface of Taper-2 having air as sensing medium.

whenever the phase matching condition is satisfied. As the Fresnel reflection formulae are valid for both planar and non-planar structures, here the simulation is done for the first point of reflection on Taper glass rod. Moreover, due to the tapering, there is a possibility of the loss of light due to refraction at the uncoated glass surface whenever the condition for TIR is not satisfied. Hence it decreases the output signal which again leads to lower signal to noise ratio. That is why very few reflections are used to avoid serious light loss. However, from Equation (10), it is clear that using tapered structure it is possible to broaden the angular range because lower value of angle means more spreading of evanescent wave field into 
region adjacent to glass-metal interface [10]. Hence coupling between evanescent wave and surface plasmon wave will become stronger.

To quantify the performance of the thin film coating over Taper-1 and Taper-2, a quality factor (Q.F) can be used which is the ratio of depth of surface plasmon resonance and its full width at half maximum. If variation of Q.F with the molar ratio of $\mathrm{AgNO}_{3} / \mathrm{KOH}$ is studied then it is clear that coating rate is maximized for optimum molar ratio of $\mathrm{AgNO}_{3} / \mathrm{KOH}$. Highest silver film quality is obtained when deposition rate is maximized. Performing silver coating at room temperature will give best result because as temperature increases there is a tendency of silver film to be oxidized [20]. To prevent the oxidization of silver film over the Tapered glass rods procedure discussed in sec 3.2 has been used in order to improve the result. The small deviation from simulated curve may be attributed to the fact that during wet deposition technique the deposited grain size is smaller $(30 \sim 60 \mathrm{~nm})$ and surface produced is rather rougher compared to vacuum deposition technique [19].

\section{Conclusion}

Tapered structure plays an important role for sensing with non-planar metal-dielectric interfaces and particularly for sensing within hollow conical devices in which it is easier to insert a tapered sensing element rather than a planar one. Present work describes the preliminary investigations on the observation of the surface plasmon resonance using such type of non planar taper structures. Further improvement of the results using sophisticated laboratory environment and precise thickness monitoring of the metal coating using vacuum deposition technique is possible and will be our next goal for future communication.

\section{Acknowledgements}

The author S. Ghosh is grateful to the Council of Scientific and Industrial Research (CSIR), India for providing the Senior Research Fellowship and K. Brahmachari is grateful to Centre of Nanoscience and Nanotechnology (CRNN), University of Calcutta for providing the grant for the execution of this work.

\section{REFERENCES}

[1] L. Novotony and B. Hecht, "Principles of Nano-Optics," Cambridge University Press Publishing, Cambridge, 2006.

[2] C.-W. Linn, K.-P. Chen, M.-C. Su, C.-K. Lee and C.-C. Yang, "Bio-Plasmonics: Nano/Micro Structure of Surface Plasmon Resonance Devices for Biomedicine," Optical and Quantum Electronics, Vol. 37, No. 13-15, 2005, pp. 1423-1437. doi:10.1007/s11082-005-4222-5

[3] H. Raether and E. Kretschmann, "Radiative Decay of
Non-Radiative Surface Plasmons Excited by Light," Zeitschrift Für Naturforschung, Vol. 23, No. 12, 1968, pp. 2135-2136.

[4] A. Otto, "Excitation of Surface Plasma Waves in Silver by the Method of Frustrated Total Reflection," Zeitschrift Für Physik a Hadrons and Nuclei, Vol. 216, No. 4, 1968, pp. 398-410. doi:10.1007/BF01391532

[5] B. Liedberg, C. Nylander and I. Sundstrom, "Surface Plasmon Resonance for Gas Detection and Biosensing," Sensors and Actuators, Vol. 4, 1983, pp. 299-304. doi:10.1016/0250-6874(83)85036-7

[6] W.-C. Kuo, C. Chou and H.-T. Wu, "Optical Heterodyne Surface-Plasmon Resonance Biosensor," Optical Letters, Vol. 28, No. 15, 2003, pp. 1329-1331. doi:10.1364/OL.28.001329

[7] S. K. Srivastava, R. K. Verma and B. D. Gupta, "Theoretical Modeling of a Localized Surface Plasmon Resonance Based Intensity Modulated Fiber Optic Refractive Index Sensor," Applied Optics, Vol. 48, No. 19, 2009, pp. 3796-3802. doi:10.1364/AO.48.003796

[8] B. D. Gupta and R. K. Verma, "Surface Plasmon Resonance-Based Fiber Optic Sensors: Principle, Probe Designs, and Some Applications," Journal of Sensors, Vol. 2009, Article ID 979761. doi:10.1155/2009/979761

[9] Y. S. Dwivedi, A. K. Sharma and B. D. Gupta, "Influence of Design Parameters on the Performance of a Surface Plasmon Sensor Based Fiber Optic Sensor," Plasmonics, Vol. 3, No. 2-3, 2008, pp. 79-86. doi:10.1007/s11468-008-9057-z

[10] R. K. Verma, A. K. Sharma and B. D. Gupta, "Surface Plasmon Resonance Based Tapered Fiber Optic Sensor with Different Taper Profiles," Optics Communications, Vol. 281, No. 6, 2008, pp. 1486-1491. doi:10.1016/j.optcom.2007.11.007

[11] R. Jha, R. K. Verma and B. D. Gupta, "Surface Plasmon Resonance-Based Tapered Fiber Optic Sensor: Sensitivity Enhancement by Introducing a Teflon Layer between Core and Metal Layer," Plasmonics, Vol. 3, 2008, pp. 151-156. doi:10.1007/s11468-008-9058-y

[12] Y. X. A. Cottenden and N. B. Jones, "A Theoretical Evaluation of Fiber-Optic Evanescent Wave Absorption in Spectroscopy and Sensors," Optics and Lasers in Engineering, Vol. 44, No. 2, 2006, pp. 93-101. doi:10.1016/j.optlaseng.2005.05.003

[13] S.-F. Wang, M.-H. Chiu and R.-S. Chang, "New Idea for a D-Type Optical Fiber Sensor Based on Kretschmann's Configuration," Optical Engineering, Vol. 44, No. 3, 2005, pp. 1-2. doi:10.1117/1.1869515

[14] M. Bera and M. Ray, "Precise Detection and Signature of Biological/Chemical Samples Based on Surface Plasmon Resonance (SPR)," Journal of Optics, Vol. 38, No. 4, 2009, pp. 232-248. doi:10.1007/s12596-009-0021-x

[15] M. Bera and M. Ray, "Multi-Layer Thin Film Modeling for Observation of Coupled Waveguide-Surface Plasmon Resonance," International Symposium on Advances in Nanomaterials (ANM2010), Kolkata, 6-7 December 2010.

[16] K. Bramhachari, S. Ghosh and M. Ray, "Application of Admittance Loci Method in Surface Plasmon Resonance 
Technology for Sensing of Different Chemical and Biological Samples," International conference on Specialty Glass \& Optical Fiber: Materials, Technology \& Devices, Kolkata, 4-6 August 2011.

[17] K. Bramhachari, S. Ghosh and M. Ray, "Experimental Observation of Surface Plasmon Resonance Using Various Geometrical Configurations of Metal-Dielectric Interface," International Symposium on Advances in Nanomaterials, Kolkata, India, 6-7 December 2010 .

[18] S. Ghosh, K. Brahmachari and M. Ray, "Experimental Investigation of Surface Plasmon Resonance Using a Chemically Deposited Silver Film on a Tapered Cylindrical Glass Rod," International conference on Speciality
Glass \& Optical Fiber: Materials, Technology \& Devices, Kolkata, 4-6 August 2011.

[19] D. S. Smith, R. S. Sathish, Y. Kostov and G. Rao, "Solution Deposition of Nanometer Scale Silver Films as an Alternative to Vapor Deposition for Plasmonic Excitation," Thin Solid Films, Vol. 518, No. 14, 2010, pp. 37723777. doi:10.1016/j.tsf.2009.12.090

[20] J. Boehm, A. Francois, H. Ebendroff-Heidopriem and T. M. Monro, "Chemical Deposition of Silver for the Fabrication of Surface Plasmon Micro Structured Optical Fiber Sensors," Plasmonics, Vol. 6, No. 1, 2011, pp. 133-136. doi:10.1007/s11468-010-9178-Z 